\title{
Raman piezospectroscopic evaluation of intergrowth ferroelectric polycrystalline ceramic in biaxial bending configuration
}

\author{
Wanyin Ge, Wenliang Zhu, Marco Deluca, and Keshu Wan \\ Ceramic Physics Laboratory \& Research Institute for Nanoscience, RIN, Kyoto Institute of Technology, \\ Sakyo-ku, Matsugasaki, 606-8585, Kyoto, Japan \\ Zhiguo Yi and Yongxiang Li \\ State Key Laboratory of High Performance Ceramics and Superfine Microstructure, Shanghai Institute \\ of Ceramics, Chinese Academy of Sciences, Shanghai 200050, People's Republic of China \\ Giuseppe Pezzotti ${ }^{\text {a) }}$ \\ Ceramic Physics Laboratory \& Research Institute for Nanoscience, RIN, Kyoto Institute of Technology, \\ Sakyo-ku, Matsugasaki, 606-8585, Kyoto, Japan
}

(Received 23 May 2006; accepted 10 October 2006; published online 1 February 2007)

\begin{abstract}
The piezospectroscopic (PS) effect was studied in an intergrowth bismuth layer-structure ferroelectric ceramic $\mathrm{Bi}_{5} \mathrm{TiNbWO}_{15}$ according to a micro-Raman spectroscopic evaluation. By using a ball-on-ring flexure configuration, a biaxial stress was generated in a $\mathrm{Bi}_{5} \mathrm{TiNbWO}_{15}$ plate-like specimen and in situ collected Raman spectra were acquired and analyzed under several loading conditions. As the observed spectral line contained signals arising from the whole illuminated in-depth region, the laser probe information was deconvoluted (by means of an in-depth probe response function obtained according to the defocusing method) in order to deduce biaxial PS coefficients for the three Raman bands of $\mathrm{Bi}_{5} \mathrm{TiNbWO}_{15}$ located at 763, 857, and $886 \mathrm{~cm}^{-1}$, respectively. The biaxial PS coefficients of these bands were derived to be $-1.74 \pm 0.16$, $-2.51 \pm 0.16$, and $-2.64 \pm 0.31 \mathrm{~cm}^{-1} / \mathrm{GPa}$, respectively, and should be referred to the $c$ axis of the Bi $_{5}$ TiNbWO $_{15}$ crystal. (C) 2007 American Institute of Physics. [DOI: 10.1063/1.2402586]
\end{abstract}

\section{INTRODUCTION}

In recent years, intergrowth bismuth layer-structure ferroelectric (IBLSF) ceramics have attracted significant interest because of the potential application of these materials in information technology, such as Ferroelectric Random Access Memory and high temperature accelerator meters. ${ }^{1} \mathrm{Al}$ though these new materials may suffer from relatively low dielectric and ferroelectric properties, potentially they represent a new generation of functional ceramics ${ }^{2-6}$ given their lead-free characteristics. In other words, their importance will be increasing as far as safety concerns related to leadbased piezoceramics will develop into rules and regulation that will further limit their application. ${ }^{7}$ Moreover, the intergrowth structure of IBLSF attracts the interest of scientists that intend to theoretically draw the origin of ferroelectricity from a structural viewpoint. ${ }^{8,9}$ Nowadays, studies on IBLSF mainly focus on the progress of their ferroelectric properties (in particular, on the development of higher electromechanical coupling and dielectric constant) and on intergrowth structure research. Unfortunately, little interest has been directed so far toward the mechanical properties, and almost no information is available in the literature on internally developed residual stress fields. In general, mechanical stress plays an important role on the reliability of microelectronic devices and it is responsible for a large variety of issues in ceramic technology, such as toughness enhancement, ${ }^{10}$ slow crack growth, ${ }^{11,12}$ and environmentally assisted aging. ${ }^{13}$ Re-

\footnotetext{
${ }^{a)}$ Author to whom correspondence should be addressed; electronic mail: pezzotti@kit.ac.jp
}

sidual stress has a profound effect on the materials properties, e.g., electrical (Hall effect, dielectric relaxation, electromobility, etc.); optical (band gap, optical emission, etc.); and other properties as ferroelectricity and ferroelasticity. In order for IBLSF to be widely considered in practical applications, the effect of mechanical stress must be taken into account, which will be introduced during processing of the material itself or device manufacturing.

Residual stress fields in ferroelectrics have been measured by x-ray diffraction (XRD) methods, but the growing trend toward smaller-scale devices pushes toward the development of high-spatial resolution techniques. Raman microprobe piezospectroscopy (PS) is a well-known analytical method for residual stress assessment in ceramics and it has been applied to ferroelectrics not only from a fundamental point of view, but also for the analysis of domain orientation $^{14,15}$ and for stress assessments in devices. ${ }^{16}$ The main advantages of Raman spectroscopy, compared to other techniques, are the nondestructive nature of the evaluation, its high spatial resolution (down to $1 \mu \mathrm{m}$ in the lateral extent), and its suitability for in situ investigations.

In this article, we aim to investigate the PS effect in IBLSF $\mathrm{Bi}_{5} \mathrm{TiNbWO}_{15}$ by using microprobe Raman spectroscopy. Using a known stress state, generated in ball-on-ring bending configuration of the ceramic plate, the biaxial PS coefficients of the main Raman bands of $\mathrm{Bi}_{5} \mathrm{TiNbWO}_{15}$ ceramic could be determined. Moreover, the laser probe geometry was quantitatively studied by considering the probe/ sample interaction. The probe contribution to the signal was evaluated and an in-depth deconvolution procedure was ap- 
plied in order to reduce the error introduced by the finite size of the illuminated area. This study provides the backbone to future work on the quantitative assessment of unknown residual stress fields and reliability of intergrowth lead-free ferroelectric devices.

\section{THEORETICAL BACKGROUND AND EXPERIMENTAL PROCEDURES}

\section{A. Piezospectroscopy and related assessments}

The PS effect is defined as the shift of the Raman or fluorescence spectrum of a solid in response to an applied stress or strain. ${ }^{17,18}$ The origin of the PS effect is the change of the energy of the electron or phonon transition state in the solid due to the applied stress. ${ }^{19}$ From a theoretical viewpoint, the relationship between the spectral shift and the stress can be described by a Taylor's series expansion, as follows:

$$
\omega=\omega_{0}+\left(\frac{\delta \omega}{\delta \sigma_{i j}}\right)_{i j}^{0} \sigma_{i j}+\frac{1}{2}\left(\frac{\delta^{2} \omega}{\delta \sigma_{i j} \delta \sigma_{k l}}\right)_{i j k l}^{0} \sigma_{i j} \sigma_{k l}+\cdots,
$$

where $\omega_{0}$ refers to the band center of the spectrum at zero stress, and $\sigma_{i j}$ is the applied stress tensor. Upon neglecting terms higher than the first-order one, Eq. (1) reduces to

$$
\frac{\omega-\omega_{0}}{\sigma_{i j}}=\left(\frac{\delta \omega}{\delta \sigma_{i j}}\right)_{i j}^{0} \text {. }
$$

Therefore, the relationship between the shift of the spectral band center of the materials and the applied stress is the first-order coefficient of Taylor's series and can be expressed with the symbol $\Pi_{i j}$, with Eq. (1) rewritten in the following form:

$$
\frac{\Delta \omega}{\sigma_{i j}}=\prod_{i j}
$$

It should be noted that Eq. (3) is a tensorial equation and $\Pi_{i j}$ is a second-rank tensor. This tensor can be simplified greatly if the principal stresses are applied along known crystallographic directions. ${ }^{20}$ From Eq. (3), an unknown mean stress magnitude can be determined by measuring the shift of the spectrum when the components of the second-rank PS tensor are known. Inversely, the PS coefficients can be determined from the observed spectral shift versus stress magnitude when a known stress state (hydrostatic, uniaxial, or biaxial) is applied to the material. If it is possible to make assumptions on the material microstructure Eq. (3) can be significantly simplified; e.g., for isotropic materials, the trace of the stress tensor can be directly measured.

In the case of a biaxial stress field, several flexure configurations, such as the ball-on-ring, the ring-on-ring, and the piston-on-ring configurations, are available. ${ }^{21-25}$ Among them, the ball-on-ring flexure, which has been developed for more than 40 years, ${ }^{26}$ has become a common tool for stress measurements in thin films. This flexure jig is especially useful to analyze plate-like samples such as ceramic wafers and thin films, because (i) a biaxial stress is often responsible for their failure and (ii) the sample size required for the analysis

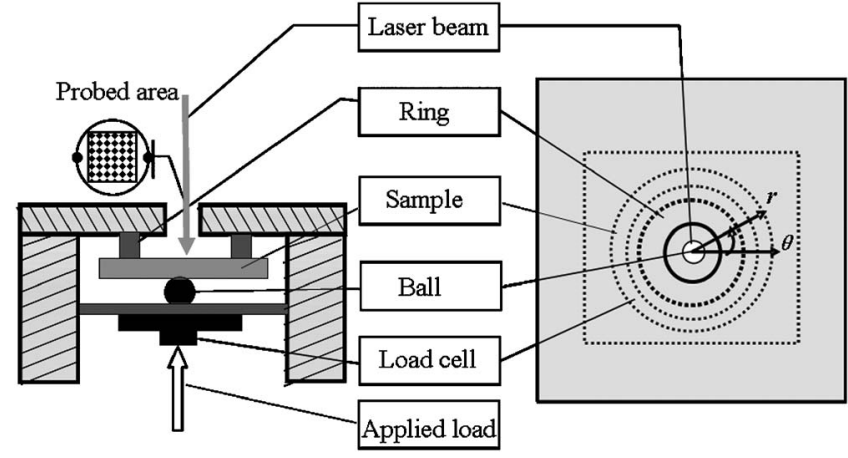

FIG. 1. Structural configuration of the ball-on-ring jig.

(especially the thickness) can be very small compared to other flexure methods such as uniaxial bending, which needs relative bigger and long bar specimen.

A number of studies have illustrated that this type of flexure is accurate and its reliability has been proved by both experimental work and theoretical calculations. ${ }^{21,24,26}$

Figure 1 shows the main features of the ball-on-ring jig configuration adopted in this study. The specimen is located between the metal ring and the ball within the jig and the load is monitored by a load cell.

A radial stress $\sigma_{r}$ and a tangential stress $\sigma_{t}$ are introduced on the surface of the specimen when the specimen is loaded. $^{27}$ The maximum radial stress $\sigma_{r}$ and tangential stress $\sigma_{t}$ are equal at the center of the ring

$$
\sigma_{r}=\sigma_{t}=\sigma_{\max }=\frac{3 P(1+\nu)}{4 \pi t^{2}}\left(1+2 \ln \frac{D}{d}+\frac{1-\nu}{1+\nu} \frac{2 D^{2}-d^{2}}{4 R^{2}}\right),
$$

where $P$ is the applied load, $\nu$ is the Poisson's ratio of the material, $t$ is the thickness of the plate specimen, $D$ is the diameter of the ring, and $R$ is half the planar dimension of the plate specimen. $d$ is the diameter of a region of approximately uniform loading at the plate center. According to the Westergaard's equation, ${ }^{28}$ the $d$ value is a function of the plate thickness, $t$,

$$
\begin{aligned}
& d=2 t \cdot\left(d_{\text {contact }}>3.488 t\right), \\
& d=\sqrt{1.6 d_{\text {contact }}^{2}+4 t^{2}}-1.35 t \cdot\left(d_{\text {contact }}<3.488 t\right), \\
& d=0.65 t \cdot\left(d_{\text {contact }} \rightarrow 0\right),
\end{aligned}
$$

where $d_{\text {contact }}$ is the contact diameter of the loading ball with the specimen. $d_{\text {contact }}$ can be obtained from the Hertzian elastic contact stress equation between a plate and a ball ${ }^{28}$

$$
\begin{aligned}
& d_{\text {contact }}=\left(\frac{3 P d_{\text {ball }}}{\chi}\right)^{1 / 3}, \\
& \chi=\left(\frac{1-\nu_{1}^{2}}{E_{1}}+\frac{1-\nu_{2}^{2}}{E_{2}}\right)^{-1},
\end{aligned}
$$

where $d_{\text {ball }}$ is the diameter of the loading ball, $\nu$ is Poisson's ratio, $E$ is Young's modulus, and the subscripts 1 and 2 denote the plate and the loading ball, respectively. 
Within a central region of diameter $d$, the stress variation is very small and the stress magnitude can be regarded as constant. However, outside this area, the stress decreases steeply along the radial direction according to the following formulas:

$$
\begin{aligned}
& \sigma_{r}=\frac{3 P(1+\nu)}{4 \pi t^{2}}\left[2 \ln \frac{D}{2 r}+\frac{1-\nu}{2(1+\nu)} \frac{D^{2}-4 r^{2}}{4 R^{2}} \frac{d^{2}}{4 r^{2}}\right], \\
& \sigma_{t}=\frac{3 P(1+\nu)}{4 \pi t^{2}}\left[2 \ln \frac{D}{2 r}+\frac{1-\nu}{2(1+\nu)} \frac{D^{2}}{4 R^{2}}\left(4-\frac{d^{2}}{4 r^{2}}\right)\right] .
\end{aligned}
$$

Note that $r$ is the radial distance with origin at the center of the ring. In this case, the radial stress $\sigma_{r}$ and the tangential stress $\sigma_{t}$ are independent of the angular orientation along the radial direction. For a certain given point, the biaxial stress magnitude can be calculated by summing up the magnitude of radial and tangential stress, that is

$$
\sigma_{r}+\sigma_{t}=\frac{3 P(1+\nu)}{4 \pi t^{2}}\left[4 \ln \frac{D}{2 r}+\frac{1-\nu}{2(1+\nu)} \frac{4 D^{2}-d^{2}}{4 R^{2}}\right] .
$$

In the linear elastic field, the biaxial stress distribution along the in-depth direction, $z$, of a ceramic plate loaded in a ball-on-ring configuration is linearly related to the (maximum) surface stress according to a proportionality coefficient, $(t-2 z) / t$. Therefore, according to Eqs. (4), (10), and (11), the biaxial stress field at any location of the plate-like specimen loaded in the ball-on-ring configuration can be calculated.

\section{B. In-depth probe response function}

The observed Raman spectrum can be considered as the summation of the local spectral contribution from any individual point within the finite volume of a laser probe focused at a point $\left(x_{0}, y_{0}, z_{0}\right) .{ }^{29}$ The spatial probe response function (PRF) that describes the observed intensity can be expressed considering the intensity distribution in-plane $x, y$, lateral response and the in-depth, $z$, response, this latter giving the resolution along the laser focal axis. ${ }^{30,31}$ The complete morphology of the PRF can be given as follows:

$$
\begin{aligned}
I_{\mathrm{obs}} \propto & \iint_{S_{x y}} \int_{0}^{\infty} \frac{A^{2}}{\left(\omega-\omega_{0}\right)^{2}+A^{2}} \times \frac{p^{2}}{p^{2}+\left(z-z_{0}\right)^{2}} \\
& \times \exp \left[-2 \frac{\left(x-x_{0}\right)^{2}+\left(y-y_{0}\right)^{2}}{B^{2}}\right] d x d y d z,
\end{aligned}
$$

where $S_{x y}$ refers to the probed planar ( $x y$ plane) at any depth $z$. $A$ is the full width at half maximum of the local spectral band; $\omega_{0}$ is the center of the band; $B$ and $p$ are the half peak width of the in-plane and of the in-depth PRFs, respectively.

Equation (13) contains a three-dimensional integral that is difficult to separate into independent parts. However, for the stress distribution discussed in the present study, gradients in the $x y$ plane can be neglected, considering that the laser spot size is of a micrometric size. Accordingly, Eq. (13) can be simplified as follows:

$$
I_{\mathrm{obs}}(\omega) \propto \int_{0}^{\infty} \frac{A^{2}}{\left(\omega-\omega_{0}\right)^{2}+A^{2}} \times \frac{p^{2}}{p^{2}+\left(z-z_{0}\right)^{2}} d z .
$$

Equation (14) can be integrated analytically in case of transparent and semitransparent materials

$$
\frac{I_{\mathrm{obs}}}{I_{\mathrm{obs}, \max }}=\frac{1}{2}-\frac{1}{\pi} \arctan \left(-\frac{z_{0}}{p}\right),
$$

where $I_{\mathrm{obs} \text {,max }}$ is the maximum observed intensity of the selected band of the spectrum upon varying defocusing distance, $z_{0}$. The $I_{\mathrm{obs}} / I_{\mathrm{obs}, \max }$ is the normalized intensity of the spectrum signal along the $z$ direction and represents the indepth PRF. The probe response parameter $p$ can be determined by plotting the normalized intensity of the spectrum and considering the derivative of the curve obtained. This method enables one to characterize the amount of signal contributed by each individual plane within the irradiated volume of the probe.

When both incident and scattered/emitted light are absorbed by the specimen with an absorption coefficient, $\alpha$, the combined effects can be represented by an effective intensity at any given point. In addition, for polycrystalline ceramics the effect of grain boundaries should be considered, since refraction, deflection, and diffraction of light among grains occurs. If the grain size is small enough, the probe can contain a sufficient number of grains that averages the effect of polarization on the probe interaction, so it is possible to incorporate the effect of inhomogeneous scattering into absorption, by using an effective absorption coefficient, $\alpha_{\text {eff }}{ }^{32}$ Accordingly, the observed spectrum can be expressed by

$$
\begin{aligned}
I_{\mathrm{obs}} \propto & \iint_{S_{x y}} \int_{0}^{\infty} \frac{A^{2}}{\left(\omega-\omega_{0}\right)^{2}+A^{2}} \times \frac{p^{2}}{p^{2}+\left(z-z_{0}\right)^{2}} e^{-2} \alpha_{\mathrm{eff}} z \\
& \times \exp \left[-2 \frac{\left(x-x_{0}\right)^{2}+\left(y-y_{0}\right)^{2}}{B^{2}}\right] d x d y d z,
\end{aligned}
$$

and again, neglecting any distribution in the $x y$ plane, we obtain

$$
I_{\mathrm{obs}}(\omega) \propto \int_{0}^{\infty} \frac{A^{2}}{\left(\omega-\omega_{0}\right)^{2}+A^{2}} \times \frac{p^{2}}{p^{2}+\left(z-z_{0}\right)^{2}} e^{-2 \alpha_{\mathrm{eff} z}} d z .
$$

Unfortunately, no general solution is available for Eq. (17), so it has to be numerically solved case by case. In order to fit the collected Raman spectrum into an appropriate equation, a computer program was set up using commercially available software (Mathematica 5.0, Wolfram Research, Inc.).

\section{Materials and Raman spectroscopy equipment}

The ceramic sample was prepared by conventional solid state synthesis and sintering method whose details were given in a previous letter. 6 The sintered wafer was polished and cut into several plates $\phi 9.5 \times 1.44 \mathrm{~mm}$ in dimension. The specimen was inserted between the ball and the ring in the flexure configuration. The load was gradually applied and 
(a)

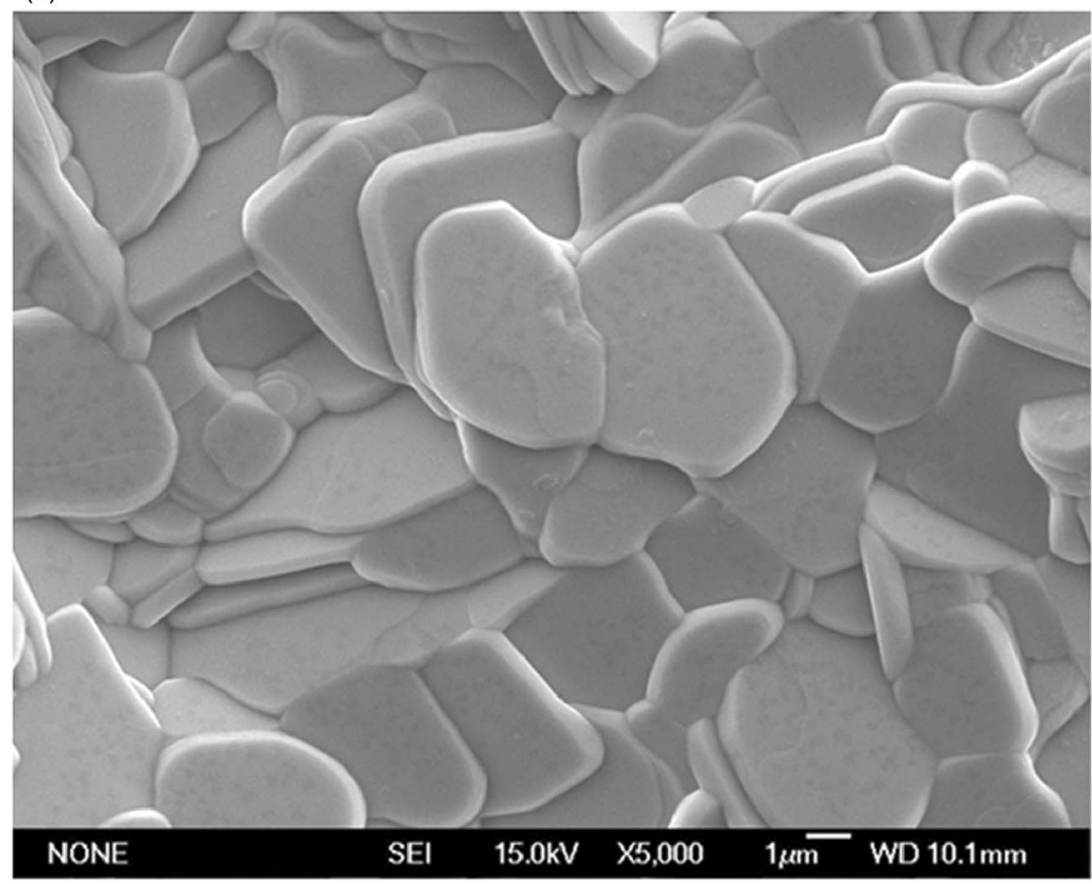

(b)

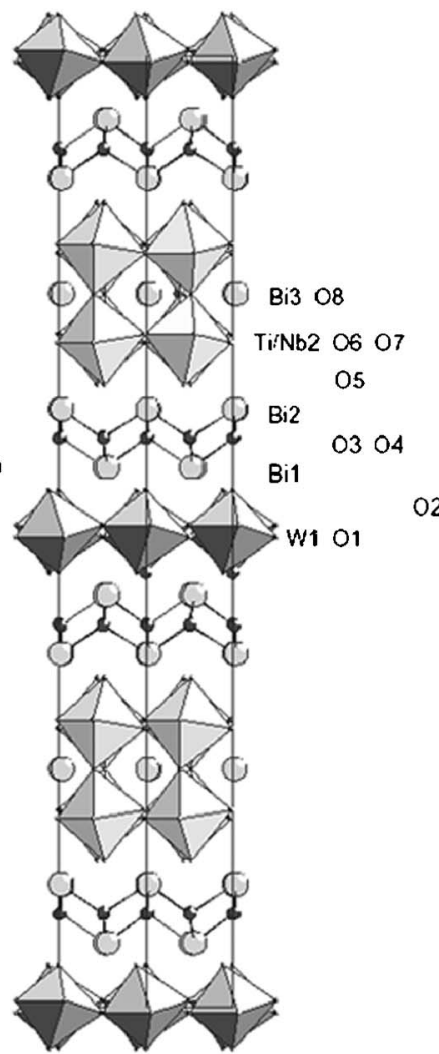

FIG. 2. (a) SEM image of polycrystalline $\mathrm{Bi}_{5} \mathrm{TiNbWO}_{15}$ ceramic. (b) Schematic draft illustrating the crystal structure of intergrowth $\mathrm{Bi}_{5} \mathrm{TiNbWO}_{15}$.

its magnitude monitored by a load cell. Raman spectra were recorded on the specimen after a steady state was reached, for every load condition.

Raman experiments were performed with a T64000 spectrometer (Horiba/Jobin-Yvon, Kyoto, Japan). The 488 $\mathrm{nm}$ wavelength of a $\mathrm{Ar}^{+}$laser (Stabilite 2017, Spectra Physics, Mountain View, CA) was used as excitation line, with a power of $400 \mathrm{~mW}$. The beam was focused at the specimen surface while mounted on the ball-on-ring jig, with a $100 \times$ (numerical aperature $=0.7$ ) objective lens which allows a lateral resolution of $1 \mu \mathrm{m}$. Raman analysis was conducted in a backscattering geometry using a triple monochromator equipped with a liquid nitrogen-cooled charge coupled device camera. The specimen stage was automatically driven by the software (LabSpec. Ver. 4.02, Jobin-Yvon) in the horizontal plane $(x, y$ plane) with an available minimum step of $0.1 \mu \mathrm{m}$. Fluctuations due to residual stress fields were found at locations near grain boundaries by preliminary probing the sample with linescans on the $x, y$ plane. Therefore, spectra were acquired by choosing a single spot at a fixed position near the center of the sample in order to minimize the effect of microstructural residual stresses. The sample/probe interaction sampling was performed on the same point by manually translating the focal plane from the surface toward either above $\left(z_{0}<0\right)$ or below the sample surface $\left(z_{0}>0\right)$. Several points were considered with performing similar measurements at different locations to assess the extent of the dispersion in the values of the PS coefficient. The spectra were collected using an accumulation time of $10 \mathrm{~s}$. We also performed experiments with different laser power (in the range from 100 to $600 \mathrm{~mW}$ ) and various accumulation time and noticed only a negligible variation in peak position of spectral bands. Therefore, in our experimental conditions, heating effect and temperature gradients can be excluded. Raw spectral data were fitted with a commercially available software package (LabSpec. Ver. 4.02, Jobin-Yvon), according to Gaussian/Lorentzian mixed functions. After fitting, the Raman band maxima could be precisely retrieved. The fitting correlation factor $\mathrm{R}^{2}$ was always better than 0.99 . scanning electron microscopy (SEM) images of the sample surface were taken with a field-emission-gun scanning electron microscope (JSM-6500F, JEOL, Tokyo, Japan).

\section{RESULTS AND DISCUSSIONS}

\section{A. Microstructure and Raman spectrum of polycrystalline $\mathrm{Bi}_{5} \mathrm{TiNbWO}_{15}$}

The surface morphology of $\mathrm{Bi}_{5} \mathrm{TiNbWO}_{15}$ was analyzed by field emission-SEM [Fig. 2(a)]. The specimen was mainly composed by stacked flake-like grains with an average size of several microns. According to an XRD analysis presented elsewhere, ${ }^{33}$ the investigated $\mathrm{Bi}_{5} \mathrm{TiNbWO}_{15}$ material possesses an intergrowth structure and belongs to the space group of $P m c 2_{1}$. The lattice constants $a, b, c$ are 2.0889(8), $0.5429(5)$, and 0.5406(4) $\AA$, respectively. The intergrowth structure is shown in Fig. 2(b), which shows the structure of the material as consisting of one half unit of $\mathrm{Bi}_{2} \mathrm{WO}_{6}$ and one half unit of $\mathrm{Bi}_{3} \mathrm{TiNbO}_{9}$. In such a structure, crystal growth was quite slow along the crystallographic $c$ axis of the material. Accordingly, the grains of this material display a flakelike appearance, as can be seen from the SEM image in Fig. 2(a). Therefore, the main directions for crystal growth are the 


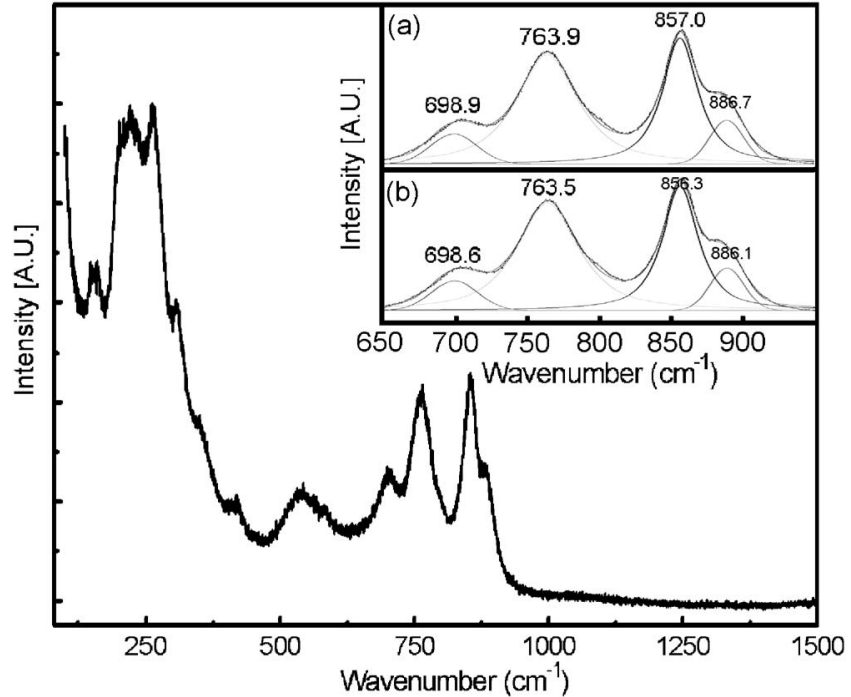

FIG. 3. Raman bands of $\mathrm{Bi}_{5} \mathrm{TiNbWO}_{15}$, the inset shows a comparison between (a) stress-free and (b) stressed (246 MPa) spectral lines. Fitting by Gaussian/Lorentzian modes is also represented.

$a$ and $b$ axes. In other words, this material can be considered as a natural superlattice structure whose $c$-plane is that observed parallel to the plane of the paper in Fig. 2. By translating this notion in terms of PS calibration, when a bending load is applied perpendicular to the disk-like sample, a biaxial stress is introduced in the $c$ plane of the crystal. Therefore, the PS coefficient obtained in this article should be considered as an average value between $a$ and $b$ planes, thus being strictly valid only for the $c$ plane of the crystal.

The Raman spectrum of the present $\mathrm{Bi}_{5} \mathrm{TiNbWO}_{15}$ sample is shown in Fig. 3. Detectable Raman bands were located at around 157, 206, 228, 267, 418, 546, 704, 763, and $857 \mathrm{~cm}^{-1}$. According to previous studies, ${ }^{33-35}$ the Raman bands can be assigned as follows: the $157 \mathrm{~cm}^{-1}$ band is associated with the vibration of the $\mathrm{Bi}^{3+}$ ions at $\mathrm{A}$ sites in perovskite-like layers along the $z$ direction (TO mode $A_{1 g}$ ). The bands between 200 and $270 \mathrm{~cm}^{-1}$ represent the rocking mode of the octahedral $\left(E_{g}\right)$. The bands between 400 and $600 \mathrm{~cm}^{-1}$ can be assigned to the opposing excursions of the external apical oxygen atoms of the octahedral $\left(E_{g}\right.$ mode). The bands at 763 and $857 \mathrm{~cm}^{-1}$ represent the symmetric stretching of the $\mathrm{WO}_{6}$ and of the $(\mathrm{Ti}, \mathrm{Nb}) \mathrm{O}_{6}$ octahedral, respectively. The $704 \mathrm{~cm}^{-1}$ as well as the right shoulder of band $857 \mathrm{~cm}^{-1}$ (located at $\sim 886 \mathrm{~cm}^{-1}$ ) are due to the orthorhombic distortion of the octahedral. The present PS characterization will be mainly focused on the Raman bands observed between 700 and $1000 \mathrm{~cm}^{-1}$ because of their strong and sharp characteristics. In the inset of Fig. 3, details are reported of a selected band in a comparison between its stress-free and stressed configurations. It is important to note that a stress gradient within the probe results in an asymmetric line shape. Therefore, the definition of peak position, and consequently of peak shift, is not unique as it is for symmetric functions, and becomes arbitrary (it could be the center of mass, the maximum, etc.). Therefore, strictly speaking, when the Raman bands are fitted with symmetric lines, error is involved. However, this effect is negligible here, as shown in

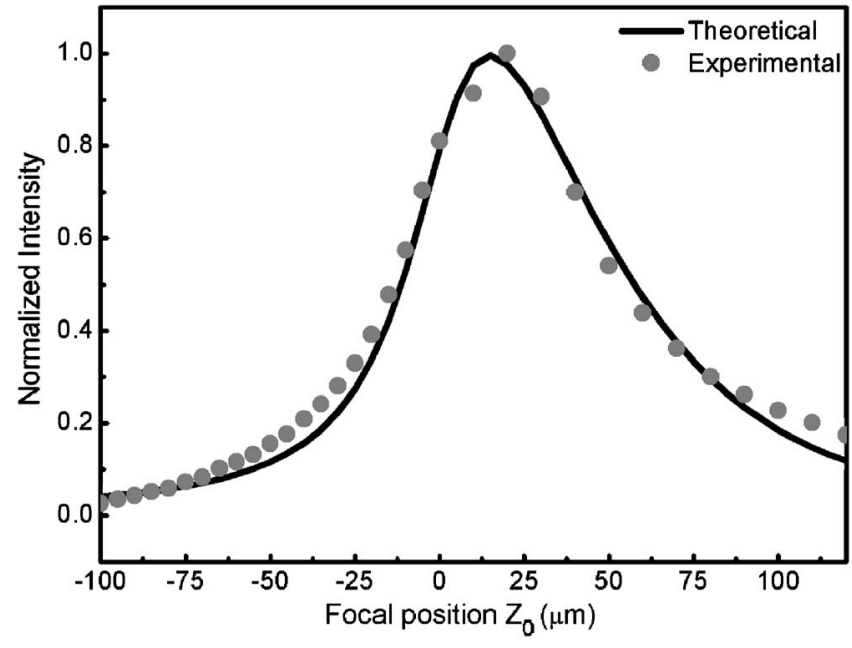

FIG. 4. Variation of the normalized intensity with defocusing position in $\mathrm{Bi}_{5} \mathrm{TiNbWO}_{15}$ ceramic.

the comparison between stressed and unstressed bands in the inset of Fig. 3. The rationale behind this argument is that the stress changes by less then $5 \%$ in the first $30 \mu \mathrm{m}$ depth of the sample.

\section{B. In-depth PRF for polycrystalline $\mathrm{Bi}_{5} \mathrm{TiNbWO}_{15}$ ceramic}

The sample/probe interaction in polycrystalline $\mathrm{Bi}_{5} \mathrm{TiNbWO}_{15}$ ceramic was analyzed according to the defocusing method (cf. Sec. II B). Figure 4 shows the defocusing behavior obtained for the $\mathrm{Bi}_{5} \mathrm{TiNbWO}_{15}$ ceramic specimen when the laser beam was initially focused on the sample surface and then the specimen stage was displaced stepwise in order to translate the focal plane from the surface either above $\left(z_{0}<0\right)$ or below the sample surface $\left(z_{0}>0\right)$.

The theoretical curve was obtained by numerically solving Eq. (17) and the values of the probe response parameter $p$ and the effective optical absorption coefficient $\alpha_{\text {eff }}$ were adjusted to give the best fit to the experimental data, retrieving the values $P=20 \mu \mathrm{m}$ and $\alpha_{\text {eff }}=0.017 \mu \mathrm{m}^{-1}$. The penetration depth $Z_{p}$ is defined as the length at which the intensity of the selected Raman band is $90 \%$ of its maximum value. $Z_{p}$ was calculated according to the following equation:

$$
\frac{\int_{0}^{Z_{p}} \frac{p^{2}}{p^{2}+\left(z-z_{0}\right)^{2}} e^{-2 \alpha_{\mathrm{eff} z} d z}}{\int_{0}^{\infty} \frac{p^{2}}{p^{2}+\left(z-z_{0}\right)^{2}} e^{-2 \alpha_{\mathrm{eff} z} d z}}=90 \% .
$$

The retrieved $Z_{p}$ value was $30 \mu \mathrm{m}$, according to the above equation, which demonstrated that $\mathrm{Bi}_{5} \mathrm{TiNbWO}_{15}$ ceramic is semi-transparent to the incident laser. Inside the material, the probe has a finite size over which the Raman signal is averaged, and thus causes deviation of the measured peak shift from the real one at the focal position, if a nonuniform stress distribution in the in-depth direction exists. Therefore, in a ball-on-ring PS calibration, which involves a stress gradient along the in-depth direction, a deconvolution 
procedure may be needed to take into account the finite size of the probe and to enable the obtainment of the actual magnitude of the PS coefficient.

\section{In-depth deconvoluted PS calibration for polycrystalline $\mathrm{Bi}_{5} \mathrm{TiNbWO}_{15}$ ceramic}

The magnitude and distribution of stress in the present ball-on-ring flexure was experimentally validated in a previous study on single crystalline silicon (100), and the Raman PS coefficient has been precisely measured for this material. ${ }^{36}$ However, since silicon has a very strong absorption for incident light with $488 \mathrm{~nm}$ wavelength, the laser penetration depth is only about $0.7 \mu \mathrm{m}$ and the contribution to the observed spectrum will mainly come from the very surface region of the sample. In other words, the in-depth stress distribution, which could be neglected in assessing the PS behavior of a silicon wafer, is likely to play a role in polycrystalline $\mathrm{Bi}_{5} \mathrm{TiNbWO}_{15}$ ceramic, whose transparency to a $488 \mathrm{~nm}$ laser is more than 40 times higher than that of silicon. $^{36}$

In addition, unlike single-crystalline silicon, the collected Raman spectrum in the present coarse-grained and textured polycrystalline $\mathrm{Bi}_{5} \mathrm{TiNbWO}_{15}$ ceramic may depend on local grain orientation. For this reason, while in the PS study of single-crystalline silicon the radial stress distribution was monitored with a line scan along an arbitrary diameter on the plate surface under a constant load, in the present study of $\mathrm{Bi}_{5} \mathrm{TiNbWO}_{15}$ material, the load was gradually increased and the Raman spectrum recorded exactly at the same locations (i.e., at the center of the plate) for each load. In the linearly elastic stress region, the biaxial stress distribution along the in-depth direction in the ball-on-ring configuration is linear and can be expressed as follows:

$$
\sigma_{z}=\frac{t-2 z}{t} \sigma_{\max },
$$

where $\sigma_{\max }$ is the biaxial stress value at the center of the ring on the sample surface [cf. Eq. (12)] and $t$ is the thickness of the plate. The biaxial stress state changes from tensile on the ring side to compressive stress on the ball side along the $z$ axis and there is a neutral surface at the center of the plate where the biaxial stress is zero. The maximum tensile and compressive stresses are located on the sample surfaces and are of the same magnitude. A schematic draft of both stress distribution in the ceramic plate and laser penetration depth is shown in Fig. 5.

If the spectral shift of the selected Raman band is significantly smaller than the respective band width [i.e., $(\omega$ $\left.-\omega_{0}\right)^{2} \ll A^{2}$ in Eq. (17)], the observed peak shift can be expressed by an average of $\omega_{0}$ over the depth $z$, weighted by the effective scattered intensity at that point. ${ }^{20,36}$ Therefore, when the laser is focused at the ring-side surface, the average values of the spectral shift, $\overline{\Delta \omega_{z}}$, in the probe volume can be expressed as follows:

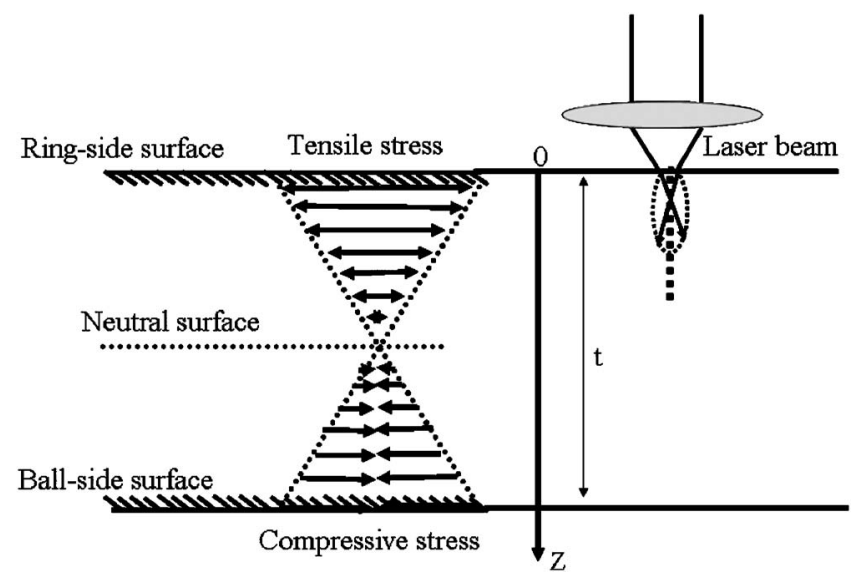

FIG. 5. Schematic draft of the stress distribution in the plate-like sample along the $z$ axis, related to the laser probe geometry.

$$
\overline{\Delta \omega_{z}}=\frac{\int_{0}^{Z_{p}} \Delta \omega_{z} \times \frac{p^{2}}{p^{2}+\left(z-z_{0}\right)^{2}} e^{-2 \alpha_{\mathrm{eff} z}} d z}{\int_{0}^{\infty} \frac{p^{2}}{p^{2}+\left(z-z_{0}\right)^{2}} e^{-2 \alpha_{\mathrm{eff} z}} d z} .
$$

According to the piezospectroscopic formalism, Eq. (20) can be solved numerically to find the deconvoluted spectral shift, $\Delta \omega_{z}$, from the observed (convoluted) shift, $\overline{\Delta \omega_{z}}$, of the selected Raman band. Note that, according to a conceptually similar approach, one could also plot the convoluted shift amount, $\overline{\Delta \omega_{z}}$ vs convoluted stress, $\bar{\sigma}_{z}$, to obtain the PS coefficient, as follows:

$$
\overline{\Delta \omega_{z}}=\Pi_{b} \overline{\sigma_{z}} \text {. }
$$

This procedure is based on the simple rationale that the spectrum will shift by the exact amount (i.e., the convoluted shift) given by the amount of stress the probe actually "feels" (i.e., the convoluted stress). Although it is clear why this can be an advantageous definition, the stress has a well defined, unique value for each $z$, and its convolution with a weighting function may have no deep physical meaning. Rather, it is the observed shift that results from the superposition of weighted contributions. Therefore, the biaxial PS coefficient, $\Pi_{b}$, was obtained from the slope of a plot of deconvoluted shift $\left(\Delta \omega_{z}\right)$ versus stress. These plots are shown in Figs. 6(a)-6(c) for the 763, 857, $886 \mathrm{~cm}^{-1}$ Raman bands of $\mathrm{Bi}_{5} \mathrm{TiNbWO}_{15}$. As seen, the PS response of each Raman band was different in $\mathrm{Bi}_{5} \mathrm{TiNbWO}_{15}$. The retrieved PS coefficients (obtained from the average of the slopes of the respective plots collected at several locations, by a least-square routine) are summarized in Table I. Note that here a PS coefficient with a negative sign means that compressive stress will cause a spectral shift toward higher wave numbers. Similar PS plots were drawn, for comparison, by using the stress values as calculated on the sample surface, with no further convolution procedure involved with assessing their magnitude, and an underestimation of about $10 \%$ in the $\Pi_{b}$ value was found, independent of the investigated Raman band. Note that the error amount related to probe convolution is just slightly higher than the experimental error affecting the PS approach (Table I). In the present bending con- 

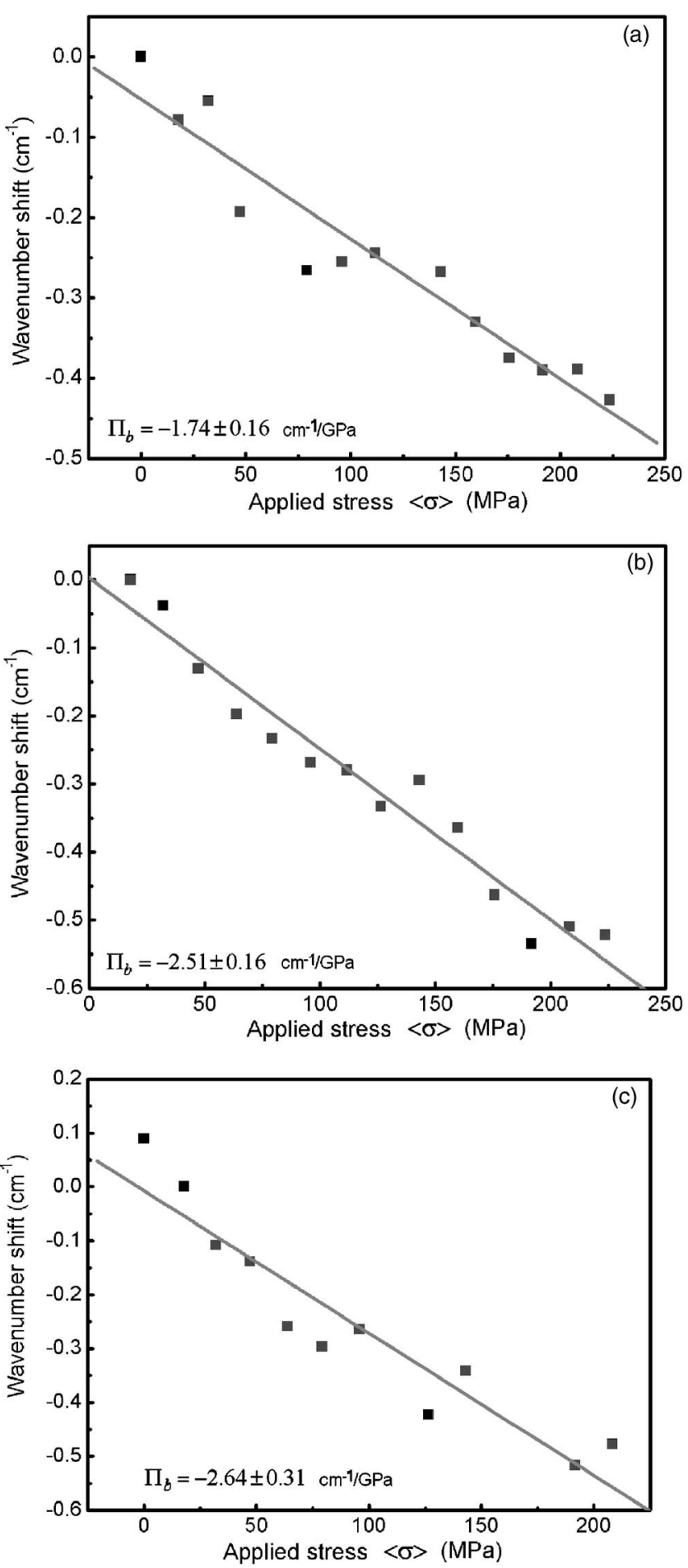

FIG. 6. Deconvoluted spectral shift vs stress for Raman bands of $\mathrm{Bi}_{5} \mathrm{TiNbWO}_{15}$ ceramic located at 763, 857, and $886 \mathrm{~cm}^{-1}$ [in (a), (b), and (c), respectively]. The average error involved in the plots is $6.5 \%$.

figuration, the stress gradient was relatively small, due to the relatively high thickness of the sample; therefore, in can be argued that the effect of the stress gradient on the Raman shift was predictably insignificant. However, our material has a relatively small absorption with respect to the incident laser which leads to a relatively large probe $\left(Z_{p} \approx 30 \mu \mathrm{m}\right)$, involving a clear stress gradient within the probe. As a result, we learned from the experiments that the convolution effect led to an error that was just slightly larger than the experimental
TABLE I. The measured biaxial PS coefficient for Raman bands of $\mathrm{Bi}_{5} \mathrm{TiNbWO}_{15}$ ceramic located at 763,857 , and $886 \mathrm{~cm}^{-1}$, respectively. The error was calculated according to the linear least square method of fitting in the PS calibration diagrams

\begin{tabular}{ccc}
\hline \hline Raman band $\left(\mathrm{cm}^{-1}\right)$ & $\Pi_{b}\left(\mathrm{~cm}^{-1} / \mathrm{GPa}\right)$ & Error $\left(\mathrm{cm}^{-1} / \mathrm{GPa}\right)$ \\
\hline 763 & -1.74 & 0.16 \\
857 & -2.51 & 0.16 \\
886 & -2.64 & 0.31 \\
\hline
\end{tabular}

error. However, in the general case of PS calibration in ballon-ring bending configuration, it is preferable to take into proper consideration probe convolution effects.

\section{CONCLUSION}

The biaxial PS coefficients of three of the most intense Raman bands of IBLSF ceramic $\mathrm{Bi}_{5} \mathrm{TiNbWO}_{15}$ were determined by Raman microprobe piezo-spectroscopy by applying a biaxial (elastic) stress state to a plate-like specimen in a ball-on-ring flexure jig. The distribution of biaxial stress field in this jig was validated in a previous study using singlecrystalline silicon prior to testing the present ferroelectric ceramic. The absorption characteristics of the $\mathrm{Bi}_{5} \mathrm{TiNbWO}_{15}$ ceramic and the laser PRF along the $z$ axis were analyzed according to the defocus method. In order to obtain the precise value of PS coefficient of the $\mathrm{Bi}_{5} \mathrm{TiNbWO}_{15}$ ceramic, a probe convolution process was adopted and the influence of the finite probe size on the monitored spectral shift, in terms of error, was found to be slightly higher than that involved with PS assessments. The biaxial PS coefficients of the 763 , 857 , and $886 \mathrm{~cm}^{-1}$ bands, were $-1.74,-2.51$, and $-2.64\left(\mathrm{~cm}^{-1} / \mathrm{GPa}\right)$, respectively. The authors confide that in light of the present contribution a nondestructive stress analysis routine will be established, which will greatly help in the actual application of intergrowth bismuth layerstructure ferroelectric $\mathrm{Bi}_{5} \mathrm{TiNbWO}_{15}$ ceramics in the ferroelectric field.

\section{ACKNOWLEDGMENT}

The authors would like to thank S. Shiroyama (Ceramic Physics Laboratory) for his help with the Raman experiments.

${ }^{1}$ C. A. P. de Araujo, J. D. Cuchiaro, L. D. McMillan, M. Scott, and J. F. Scott, Nature (London) 374, 627 (1995)

${ }^{2}$ T. Kikuchi, J. Less-Common Met. 48, 319 (1976).

${ }^{3}$ Y. Goshima, Y. Noguchi, and M. Miyayama, Appl. Phys. Lett. 81, 2226 (2002).

${ }^{4}$ J. Zhu, X. B. Chen, W. P. Lu, X. Y. Mao, and R. Hui, Appl. Phys. Lett. 83, 1818 (2003)

${ }^{5}$ A. Snedden, D. O. Charkin, V. A. Dolgikh, and P. Lightfoot, J. Solid State Chem. 178, 180 (2005).

${ }^{6}$ Z. G. Yi, Y. X. Li, Z. Y. Wen, S. R. Wang, J. T. Zeng, and Q. R. Yin, Appl. Phys. Lett. 86, 192906 (2005).

${ }^{7}$ H. Takeda, R. Aoyagi, M. Matsushita, and T. Shiosaki, Appl. Phys. A: Mater. Sci. Process. 76, 295 (2003).

${ }^{8}$ G. N. Subbanna, T. N. Guru Row, and C. N. R. Rao, J. Solid State Chem. 86, 206 (1990).

${ }^{9}$ R. L. Withers, J. G. Thompson, and A. D. Rae, J. Solid State Chem. 94, 404 (1991).

${ }^{10}$ A. V. Virkar, J. L. Huang, and R. A. Cutler, J. Am. Ceram. Soc. 70, 164 (1987). 
${ }^{11}$ J. A. Connally and S. B. Brown, Science 256, 1537 (1992).

${ }^{12}$ A. G. Evans, Int. J. Fract. 10, 251 (1974).

${ }^{13}$ V. Sergo and D. R. Clarke, J. Am. Ceram. Soc. 78, 641 (1995)

${ }^{14}$ Z. Li, C. M. Foster, X. H. Dai, X. Z. Xu, S. K. Chan, and D. J. Lam, J. Appl. Phys. 71, 4481 (1992).

${ }^{15}$ M. Deluca, C. Galassi, and G. Pezzotti, Ferroelectr., Lett. Sect. 32, 31 (2005).

${ }^{16}$ G. Pezzotti, H. Ichimaru, L. P. Ferroni, K. Hirao, and O. Sbaizero, J. Am Ceram. Soc. 84, 1785 (2001).

${ }^{17}$ R. Forman, G. Piermarini, J. Barnett, and S. Block, Science 176, 284 (1972).

${ }^{18}$ L. Grabner, J. Appl. Phys. 49, 580 (1978).

${ }^{19}$ G. Pezzotti, J. Raman Spectrosc. 30, 867 (1999).

${ }^{20}$ Q. Ma and D. R. Clarke, J. Am. Ceram. Soc. 76, 1433 (1993).

${ }^{21}$ J. E. Ritter, Jr., K. Jakus, and A. Batakis, J. Non-Cryst. Solids 38-39, 419 (1980).

${ }^{22}$ D. K. Shetty, A. R. Rosenfield, P. Mcguire, G. K. Bansal, and W. H. Duckworth, Am. Ceram. Soc. Bull. 59, 1193 (1980).

${ }^{23}$ G. de With and H. Wagemans, J. Am. Ceram. Soc. 72, 1538 (1989).
${ }^{24}$ R. Ham-Su and D. S. Wilkinson, J. Am. Ceram. Soc. 78, 1580 (1995).

${ }^{25}$ C. H. Hsueh, M. J. Lance, and M. K. Ferber, J. Am. Ceram. Soc. 88, 1687 (2005).

${ }^{26}$ F. Vitman and V. Pukh, Zavod. Lab. 29, 863 (1963).

${ }^{27}$ J. Wachtman and W. Capps, J. Mater. 7, 188 (1972).

${ }^{28}$ H. Hertz, J. Reine Angew. Math. 92, 156 (1881).

${ }^{29}$ D. M. Lipkin and D. R. Clarke, J. Appl. Phys. 77, 1855 (1995).

${ }^{30}$ A. Atkinson and S. C. Jain, J. Raman Spectrosc. 30, 885 (1999).

${ }^{31}$ A. Atkinson, S. C. Jain, and S. Webb, Semicond. Sci. Technol. 14, 561 (1999).

${ }^{32}$ W. Zhu and G. Pezzotti, Appl. Spectrosc. 59, 1042 (2005).

${ }^{33}$ Z. G. Yi, Y. X. Li, J. T. Zeng, Q. B. Yang, D. Wang, Y. Q. Lu, and Q. R. Yin, Appl. Phys. Lett. 87, 202901 (2005).

${ }^{34}$ Z. G. Yi, Y. X. Li, Y. Wang, and Q. R. Yin, Appl. Phys. Lett. 88, 162908 (2006).

${ }^{35}$ Z. G. Yi, Y. X. Li, Y. Wang, and Q. R. Yin, Appl. Phys. Lett. 88, 152909 (2006).

${ }^{36}$ K. Wan, W. Zhu, and G. Pezzotti, Meas. Sci. Technol. 17, 181 (2006). 